\title{
CLN3p Impacts Galactosylceramide Transport, Raft Morphology, and Lipid Content
}

\author{
ELENA RUSYN, TALAL MOUSALLEM, DIXIE-ANN PERSAUD-SAWIN, SARA MILLER, AND ROSE-MARY N. BOUSTANY \\ Departments of Neurobiology [E.R., T.M., D.-A.P.-S., R.-M.N.B.], Pathology [S.M.], Duke University Medical Center, Durham, North \\ Carolina 27710; Abu-Haidar Neuroscience Institute [R.-M.N.B.], American University of Beirut, 11072020, Beirut, Lebanon
}

\begin{abstract}
Juvenile neuronal ceroid lipofuscinosis (JNCL) belongs to the neuronal ceroid lipofuscinoses characterized by blindness/ seizures/motor/cognitive decline and early death. JNCL is caused by CLN3 gene mutations that negatively modulate cell growth/apoptosis. CLN3 protein (CLN3p) localizes to Golgi/Rab4-/Rab11-positive endosomes and lipid rafts, and harbors a galactosylceramide (GalCer) lipid raft-binding domain. Goals are proving CLN3p participates in GalCer transport from Golgi to rafts, and GalCer deficits negatively affect cell growth/apoptosis. GalCer/mutant CLN3p are retained in Golgi, with CLN3p rescuing GalCer deficits in rafts. Diminishing GalCer in normal cells by GalCer synthase siRNA negatively affects cell growth/ apoptosis. GalCer restores JNCL cell growth. WT CLN3p binds GalCer, but not mutant CLN3p. Sphingolipid content of rafts/Golgi is perturbed with diminished GalCer in rafts and accumulation in Golgi. CLN3deficient raft vesicular structures are small by transmission electron microscopy, reflecting altered sphingolipid composition of rafts. CLN1/ CLN2/CLN6 proteins bind to lysophosphatidic acid/sulfatide, CLN6/ CLN8 proteins to GalCer, and CLN8 protein to ceramide. Sphingolipid composition/morphology of CLN1-/CLN2-/CLN6-/CLN8- and CLN9deficient rafts are altered suggesting changes in raft structure/lipid stoichiometry could be common themes underlying these diseases.

(Pediatr Res 63: 625-631, 2008)
\end{abstract}

$\mathrm{T}$ he Neuronal Ceroid Lipofuscinoses (NCL)/Batten disorders are pediatric diseases characterized by retinitis pigmentosa/seizures/mental and motor decline. The pathologic hallmark of NCL is neuronal loss. Pathophysiological theories include increased lipid peroxidation, altered dolichol turnover, increased inflammation, subunit c of mitochondrial ATP synthase accumulation, and neuronal apoptosis (1).

Juvenile neuronal ceroid lipofuscinosis (JNCL) is caused by CLN3 gene mutations (2). Most cases are due to a 1.02 $\mathrm{kb}$ genomic DNA deletion, resulting in truncated protein. CLN3p has potential glycosylation, phosphorylation, and farnesylation sites. Two conserved amino acid stretches within exons 11/13, and two glycosylation sites are necessary for antiapoptotic function (2). Membrane topology suggests CLN3p has 5 transmembrane domains with extracellular/intraluminal amino- and cytoplasmic/carboxy termini (3).

Received October 25, 2007; accepted January 25, 2008.

Correspondence: Rose-Mary N. Boustany, M.D., Abu-Haidar Neuroscience Institute, American University of Beirut, Saab Medical Library Building 3rd Floor, Bliss Street, Hamra District, Beirut, Lebanon; e-mail: rb50@aub.edu.lb

This work was supported by NINDS grant RO-1 N0433344 (to R.-M.N.B) and a grant from the Batten Disease Support and Research Association (to R.-M.N.B.).

D.-A.P.-S.'s current address is NIEHS/NIH, Neurobiology/Neurotoxicology Group, III TW Alexander Drive, MD C1-04, RTP, NC 27709, USA.
CLN3p is highly conserved. Causes of cell death in the brain/retina remain controversial. CLN3p imparts antiapoptotic properties to neurons/cells (4) and regulates autophagy (5). CLN3-deficient cells have accelerated apoptosis and altered ceramide/sphingomyelin levels that correct with the addition of CLN3p. CLN3 mRNA/protein are overexpressed in cancer cells (6).

CLN3p is placed in HEK/HeLa endosomes (7), yeast vacuoles, mouse retinal cell mitochondria (8), neuronal synaptosomes/synapses (9), nucleii of rat neurons (10), and Golgi/ plasma membrane (PM) in HeLa/fibroblasts/COS-7 cells (11). Wild-type (WT) CLN3p localizes to Golgi/PM and traffics via early recycling Rab4/Rab11-endosomes from Golgi to lipid rafts (LR) in primary rat hippocampal neurons/postmitotic human neurons and fibroblasts. Mutant CLN3p localizes to a disrupted Golgi, fails to reach PM and mislocalizes to lysosomes. CLN3p harbors a conserved motif, 291VYFAE295, necessary for cell growth/apoptosis embedded in a stretch of amino acids structurally homologous to a GalCer lipid raftbinding domain. This domain defines a raft-binding site structurally identical in infectious prionic protein/ $\beta$-amyloid/V3 loop of the HIV-1 surface envelope glycoprotein, gp120 (12). Immunolabeling studies localize WT CLN3p with alkaline phosphatase/caveolin-1 to rafts and caveolae in some cells (11). Mutant CLN3p minimally colocalizes to LRs. CLN3p rescues the PM GalCer deficit suggesting involvement in GalCer transport to PM/LRs.

Rafts are involved in protein trafficking/protein-complex formation/signal transduction/apoptosis/cell adhesion/stress responses/cytoskeleton regulation/conduction of proatherogenic stimuli, and affect immune function (13-15). Rafts are portals of entry for toxins/viruses/bacteria $(16,17)$, are important for normal synapse density/morphology, and myelin integrity/myelin-axon interactions (18). Composition of HIV membranes resembles lipid microdomains supporting raft existence in cells (16). Rafts are liquid-ordered microdomains insoluble in nonionic detergents and derive from Golgi, consist of glycosphingolipids/cholesterol and are enriched in gly-

Abbreviations: ABC transporters, ATP-binding cassette transporters; CLN3, CLN3 gene; CLN3p, CLN3 protein; GalCer, galactosylceramide; GlcCer, glucosylceramide; GLTP, glycolipid transfer proteins; GRASP65, Golgi reassembly stacking protein; JNCL, Juvenile Neuronal Ceroid Lipofuscinosis; NCL, Neuronal Ceroid Lipofuscinosis; PM, plasma membrane; RT, room temperature 
cosylphosphatidylinositol/GPI-anchored proteins (19). They harbor proapoptotic ceramide (20). Prenylated CLN3p promotes association to LRs (21). These house caspase-8 activated in apoptotic CLN3-deficient cells (22). It has been suggested CLN3p contributes to [Delta]-9 desaturase activity, which targets LR-associated palmitoylated proteins (23).

CLN3p binding to GalCer in normal cells, and retention of GalCer in Golgi with paucity in rafts of CLN3-deficient cells is demonstrated. CLN3-deficient rafts exhibit altered sphingolipid composition/ultrastructure. When combined, findings suggest that WT CLN3p plays a role in transport of GalCer from endoplasmic reticulum or ER/Golgi to rafts, which is impaired in JNCL. Morphologic/biochemical changes are observed in CLN1-/CLN2-/CLN6-/CLN8-/CLN9-deficient cells, suggesting that perturbed structure/function of rafts may be a theme common to these NCLs.

\section{METHODS}

Cell culture. Lymphoblasts are grown at $37^{\circ} \mathrm{C} / 5 \% \mathrm{CO}_{2} / \mathrm{RPMI} 1640$ (Sigma Chemical Co., Aldrich, St. Louis, MO)/10\% fetal bovine serum and $1 \%$ penicillin/streptomycin/Fungizone. Immortalized lymphoblasts from JNCL/ other NCL patients/normal donors and fibroblasts from normal/mnd (CLN8deficient) mice are used. JNCL lymphoblasts are homozygous for the $1.02 \mathrm{~kb}$ deletion.

All cell lines were derived from patient blood and/or skin after obtaining consent according to a Duke University Medical Center IRB consent form and protocol.

Antibodies. Antibodies previously characterized are rabbit polyclonal antiCLN3 antibody to residues 58-77 $(10,18)$, sheep polyclonal anti-CLN6 antibody to residues 284-301 and sheep polyclonal anti-CLN8 antibody to residues 2-19, rabbit polyclonal anti-CLN2 antibody (Orbigen Inc., San Diego, CA), rabbit polyclonal anti-CLN1 antibody is also previously characterized.

GalCer distribution in fibroblasts. Reagents/Antibodies: anti-cathepsin D (Santa Cruz Biotechnology), anti-GRASP65/mouse monoclonal, anti-Rab4/ anti-Rab7/anti-Rab11/goat polyclonal, anti-alkaline phosphatase/anticaveolin-1 (RDI Concord, MA); FFA-BSA (Sigma Chemical Co.); DMEM/ FBS (Invitrogen Carlsbad, CA). Boron dipyrromethene difluoride-GalCer (BODIPY-GalCer), Alexafluor secondary antibodies, 568-red/488-green are from Molecular Probes (Eugene, OR). Normal/JNCL fibroblasts grown on poly-D-lysine-treated coverslips overnight to $80 \%$ confluence in DMEM/ $10 \% \mathrm{FBS} / 1 \%$ antibiotics $/ 37^{\circ} \mathrm{C} / 5 \% \mathrm{CO}_{2}$. Cells are incubated with BODIPYGalCer/FFA-BSA for $5 \mathrm{~min}$ at $37^{\circ} \mathrm{C} / 5 \% \mathrm{CO}_{2}$ then washed. Following the wash, cells are rinsed $3 \times$ in FFA-BSA before fixing then blocked in 5\% FFA-BSA for $1 \mathrm{~h}$ at room temperature (RT), incubated with primary antibody for $1 \mathrm{~h} / \mathrm{RT}$, washed $3 \times$ in block solution for $10 \mathrm{~min} / \mathrm{RT}$. The secondary Ab is added for $1 \mathrm{~h} / \mathrm{RT}$, slides washed $2 \times$ in blocking solution then three washes at RT/PBS and mounted on slides with Fluoromount $\mathrm{G}$ and visualized with a Zeiss LSM 510 confocal microscope (100× magnification). For the proteinlipid overlay assay Membrane arrays (SphingoStrips, Echelon Research Laboratories Salt Lake City, UT), or lipids are spotted on nitrocellulose membranes, dried then blocked with 3\% (wt/vol) FFA-BSA in Tris-Buffered Saline Tween-20 (TBST) [150 mM NaCl/10 mM Tris- $\mathrm{HCl}(\mathrm{pH} 8.0)$, and $0.1 \%$ (vol/vol) Tween-20] for $1 \mathrm{~h} / \mathrm{RT}$. Membranes are incubated for $8 \mathrm{~h} / 4^{\circ} \mathrm{C}$ with $2 \mathrm{mg} / \mathrm{mL}$ cell lysate. Fibroblasts overexpressing CLN3p/JNCL fibroblasts are used for the GalCer-CLN3p binding assay. Membranes are washed $5 \times 15 \mathrm{~min}$ with TBST, incubated with anti-CLN3/CLN1/CLN2/CLN6/CLN8-antibodies overnight at 1:1000 dilution, washed and incubated with horseradish peroxidase-conjugated anti-rabbit IgG goat antibody for $1 \mathrm{~h} / \mathrm{RT}$. After washing, enhanced chemiluminescence detects protein-sphingolipid binding.

Subcellular fractionation. Lymphoblasts are washed lysed in $300 \mu \mathrm{L}$ ice-cold media (150 mM NaCl, $5 \mathrm{mM}$ DTT (DTT), $5 \mathrm{mM}$ EDTA, $25 \mathrm{mM}$ Tris- $\mathrm{HCl}, \mathrm{pH} 7.5$, protease inhibitors, $1 \%$ Triton X-100). Samples are mixed with $300 \mu \mathrm{L}$ cold $85 \%$ sucrose, transferred into SW41Ti tubes and overlaid with $35 \%$ to $0 \%$ sucrose. Gradient mixtures are spun at $34,000 \mathrm{rpm}$ for 18 $\mathrm{h} / 4^{\circ} \mathrm{C}$. Nine $0.5 \mathrm{~mL}$ fractions (top to bottom) are collected. Two hundred fifty microliter/fraction is used for lipid extraction/TLC and $15 \mu \mathrm{L} /$ fraction for Western blotting. Equal portions/fraction are reserved for the GalCer dot-blot.

GalCer dot-blot. A total of $0.2 \mu \mathrm{L} /$ fraction is applied to nitrocellulose and dried. Membranes are blocked with 3\% (wt/vol) FFA-BSA in TBST [150 mM $\mathrm{NaCl} / 10 \mathrm{mM}$ Tris- $\mathrm{HCl}(\mathrm{pH} 8.0$ ), and $0.1 \%$ (vol/vol) Tween-20] for $1 \mathrm{~h} / \mathrm{RT}$, then incubated for $2 \mathrm{~h} / \mathrm{RT}$ with rabbit anti-GalCer/anti-Glucosylceramide (GlcCer) primary Ab (Sigma Chemical Co., Aldrich; St. Louis, MO) at 1:1000 dilution, washed $5 \times / 5 \mathrm{~min}$ with TBST and incubated with horseradish peroxidase-conjugated anti-rabbit IgG goat antibody for $1 \mathrm{~h} / \mathrm{RT}$. After washing, blots are developed in ECL-plus reagent (Amersham Biosciences, Piscataway, $\mathrm{NJ})$.

Transfection. JNCL of $1 \times 106$ lymphoblasts are plated in 6-well plates, transfected with pGEM-CLN3/empty vector control [Lipofectamine2000 Invitrogen (Carlsbad, CA)], then harvested $48 \mathrm{~h}$ later, homogenized, and subjected to subcellular fractionation as outlined above.

TLC analysis/lipid mass measurements. Lipids are extracted from 250 $\mu \mathrm{L} /$ fraction. After methanolysis and measurement of phosphate/protein, samples are spotted on borate-impregnated TLC-plates and lipids separated with chloroform/methanol/ $\mathrm{NH}_{4} \mathrm{OH} 2.5 \mathrm{M}$ (65:35:8). A mixture of standards (Qualmix, Matreya, LLC, Pleasant Gap, PA) is used. Sphingolipids are visualized with primuline and scanned on a Typhoon-101 scanner. Quantification is accomplished with the ImageQuant program. Lipids are normalized to protein.

Gal-labeling. Normal/CLN3-deficient lymphoblasts are labeled with ${ }^{14} \mathrm{C}$ galactose (Amersham, Piscataway, NJ) for $3 \mathrm{~d}$ in glucose-free RPMI1640, harvested, and subcellular fractionation performed. Protein determination for fractions is followed by lipid extraction with carriers/base hydrolysis/acid neutralization/lipid reextraction. Dried samples are resuspended and spotted on a TLC plate. Glycosphingolipds are scraped after $2 \mathrm{~d}$. Results are expressed in counts $/ \mathrm{min} / \mu \mathrm{g}$.

Western blot. MAb (flotillin-1/GRASP65/calreticulin) confirm identity of LRs/Golgi/ER fractions, respectively. Fifteen microliter from fractions 1-5 and $5 \mu \mathrm{L}$ from fractions 6-10 are mixed with SDS-PAGE buffer, boiled, and loaded on a $12 \%$ polyacrylamide gel.

RNAi knockdown of CGT in normal human lymphoblasts. siRNA is designed using BLOCK-iT RNA Designer (Invitrogen). Plasmids $(1 \mu \mathrm{g})$ expressing siRNAs were transfected into normal lymphoblasts. Cells of $48-120 \mathrm{~h}$ posttransfection are harvested. Conditions (cell density/amount of reagent/concentration of oligonucleotide/time of harvesting) are optimized using RT-PCR. RT-PCR: RNA is isolated from normal fibroblasts transfected with the GCT/scrambled siRNA with RNeasy Mini Kit (Qiagen, Valencia, CA). Reverse transcription is carried out with SuperScript III First-StrandSynthesis System (Invitrogen). RT-PCR is performed using cDNA/Absolute QPCR SYBRGreen Fluorescence Mix (Abgene Inc., Rochester, NY) in a Bio-Rad I-Cycler. Internal controls are cyclophilin/HPRT/28S ribosomal RNA. Fold-change calculated versus untreated controls was 0.053 equaling 95\% knockdown. Cells are harvested after 7 days and stained with GalCer.

Propidium iodide (PI) staining. Equal numbers of cells transfected with GCT siRNA/scrambled siRNA, treated/untreated with $50 \mathrm{ng} / \mathrm{mL}$ of GalCer (Toronto Research Chemicals Inc., G184950) are suspended in $100 \mu \mathrm{L}$ PBS, incubated with $100 \mu \mathrm{L} / 0.5 \mathrm{mg} / \mathrm{mL}$ PI, washed, placed on slides and viewed using a Leica fluorescent microscope. Cells are harvested $144 \mathrm{~h}$ after transfection and $120 \mathrm{~h}$ after treatment with GalCer. Three hundred cells/three fields of vision were counted. Percentage of red staining-apoptotic cells/total cells is determined. The $t$ test was applied. Etoposide (VP-16, Sigma Chemical Co.) is used $(1 \mu \mathrm{g} / \mathrm{mL})$ to augment cell death.

Growth curves of JNCL cells with/without GalCer. Equal cell numbers are seeded and numbers determined with Trypan blue dye exclusion. Each time-point represents three experiments. JNCL cells are treated with vehicle/ GalCer (no treatment $/ 50$ or $100 \mathrm{ng} / \mathrm{mL}$ ).

Electron microscopy. LR fractions obtained by ultracentrifugation with iodixanol (OptiPrep; Norton, MA) are suspended with washing buffer $(25 \mathrm{mM}$ HEPES, $\mathrm{pH} 7.4,150 \mathrm{mM} \mathrm{NaCl})$ pelleted by ultracentrifugation $(20,000 \mathrm{rpm}$, $30 \mathrm{~min} / 0^{\circ} \mathrm{C}$ ), fixed with $4 \%$ glutaraldehyde, postfixed $1 \mathrm{~h}$ in $1 \%$ osmium tetroxide $\left(\mathrm{OsO}_{4}\right)$ in buffer, washed in veronal acetate, stained in uranyl acetate, dehydrated in a graded ethanol series and embedded in Poly/Bed812 (Polysciences; Warrington, PA). Thick/thin sections were prepared on a Reichert-Jung ultramicrotome (Leica, Bannockburn, IL). Thick sections ( 0.5 $\mathrm{mm}$ ) are stained with $1 \%$ toluidine blue-borax; thin sections are mounted on copper grids and double-stained with uranyl acetate/lead citrate. Grids are examined using a Philips EM410, 400, or CM 12 electron microscope (FEI; Hillsboro, OR).

\section{RESULTS}

GalCer subcellular localization. Normal/JNCL cells double-labeled with GalCer/organellar protein antibodies were visualized by confocal microscopy (Fig. 1). GalCer is present in Golgi/LRs/Rab4-/Rab11-positive endosomes (Fig. 1A) and relatively absent from lysosomes (Fig. 1B). GalCer is diminished in the PM and from Rab4-/Rab11-endosomes in JNCL 
Normal Fibroblasts
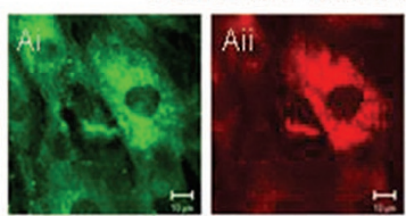

GalCer

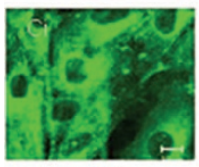

GalCer
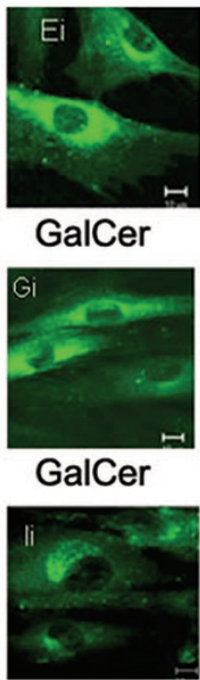

GalCer
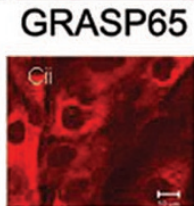

Alk. Phos.

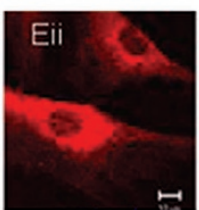

Rab4

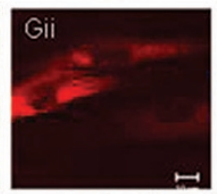

Rab11

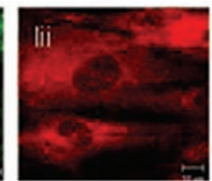

CathepsinD
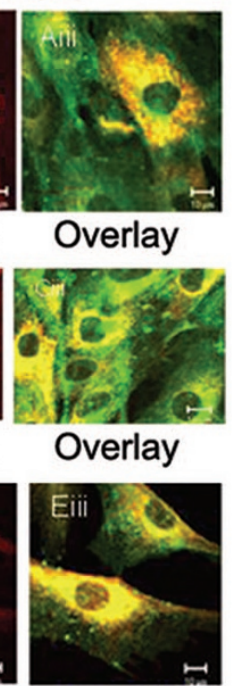

Overlay
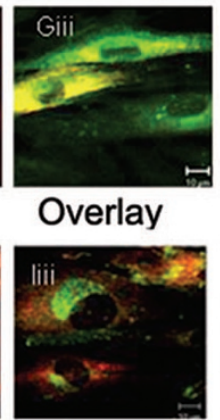

Overlay
JNLC Fibroblasts
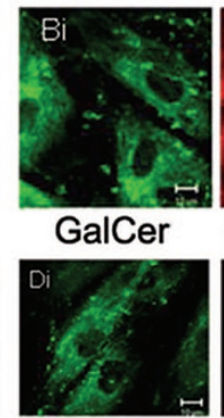

GalCer

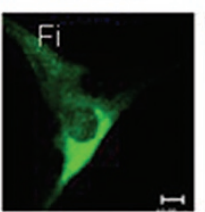

GalCer

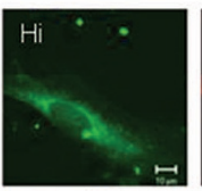

GalCer

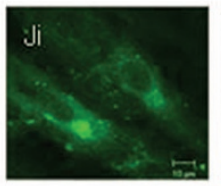

GalCer
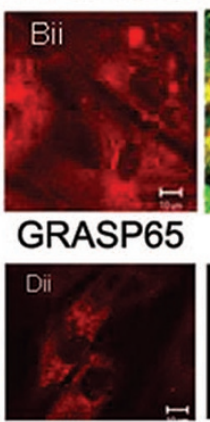

Alk. Phos.

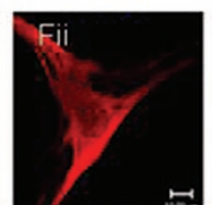

Rab4

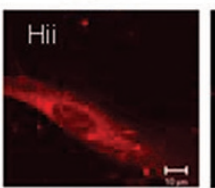

Rab11

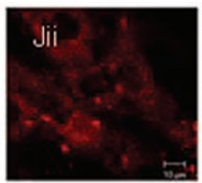

Cathepsin D

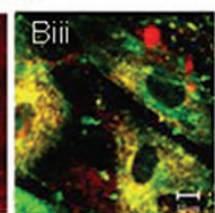

Overlay

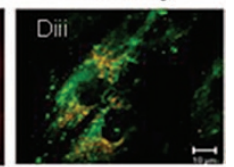

Overlay

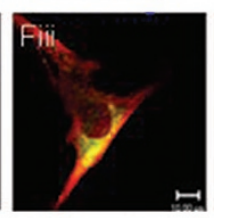

Overlay

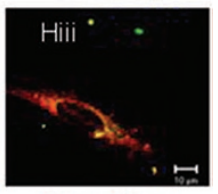

Overlay

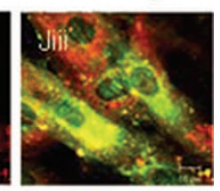

Overlay

Figure 1. GalCer localizes to Golgi/LRs/ Rab4-/Rab11-positive endosomes. NF, normal fibroblasts; JNCL, CLN3-deficient fibroblasts. ( $A, C, E$, and $G$ ) Normal fibroblasts. $(B, D, F$, and $H) \mathrm{JNCL}$ fibroblasts. $A$ and $B$ show distribution patterns for BODIPY-GalCer (green) and Golgi Reassembly Stacking protein, or GRASP65 (red). $C$ and $D$ show distribution patterns for BODIPY-GalCer (green) and Alkaline Phosphatase (red). $E$ and $F$ show distribution patterns for BODIPY-GalCer (green) and Rab4 (red). $G$ and $H$ show distribution patterns for BODIPY-GalCer (green) and Rab11 (red). $I$ and $J$ show distribution patterns of BODIPY-GalCer (green) and Cathepsin D. Regions of colocalization are yellow in overlay panels labeled (iii).

cells. GalCer colocalizes with GRASP65 in WT/JNCL cells, and the Golgi is fragmented. Golgi fragmentation is a hallmark of apoptotic neurons from these diseases: Alzheimer's disease, amyotrophic lateral sclerosis and Creutzfeldt-Jakob disease $(24,25)$. GalCer colocalizes with alkaline phosphatase, a LR marker, in $50 \%$ of normal but only $25 \%$ of JNCL cells. GalCer presence in Rab4-/Rab11-positive endosomes is confirmed by colocalization with Rab4-/Rab11 antibodies in normal, and less, in JNCL cells. This suggests that GalCer travels the same route as CLN3p. GalCer was only minimally present in lysosomes from normal fibroblasts. In JNCL cells, mutant CLN3p/GalCer localize to lysosomes as confirmed by poor but present colocalization of GalCer with Cathepsin D, a lysosomalspecific marker, in normal and strong colocalization in JNCL cells. Colocalization of CLN3p and GalCer in normal cells supports that CLN3p/GalCer trafficking are linked.

CLN3-binding to GalCer/sulfatide. Membranes impregnated with sphingolipids were overlaid with protein from CLN3-overexpressing/JNCL cells. Anti-CLN3 antibody determined binding. WT CLN3p binds strongly to sulfatide/ GalCer but mutant CLN3 does not (Fig. 2). The signal for GalCer-WT CLN3p binding was weak because polar sulfatide competes with GalCer for binding. Omitting sulfatide allowed visualization of CLN3p-binding to GalCer in a concentrationdependant manner (Fig. 2B). This supports the hypothesis that WT CLN3p may function as a GalCer transporter.
A.
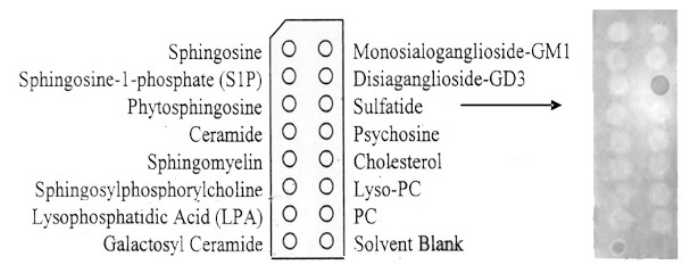

B.

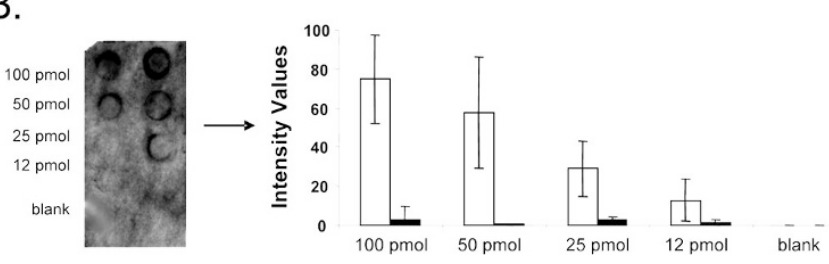

Figure 2. Lipid-protein binding assay. (A) WT CLN3p binds GalCer/ sulfatide. ( $B$ ) WT CLN3p binds GalCer in a dose-dependent manner; mutant CLN3 does not. The ImageJ program was used to compute intensity. Range of gray scale values for 8-bit images is 0 (black) to 255 (white). The drawing tool is used to select the same regions of interest (ROI) adjacent to spots with applied sphingolipids. Area of the ROI and its mean pixel intensity are measured using the "Analyze-Measure" function. The values are background-subtracted and normalized to the peak value. Relative intensity values are presented on an arbitrary scale. Results are from three experiments; error bars represent SEM. NhL ( $\square)$; JNCL (

GalCer retention in ER/Golgi of CLN3-deficient cells. Rate of synthesis and subcellular distribution of de novo GalCer/other glycosphingolipids are determined by $\left({ }^{14} \mathrm{C}\right)$ - 


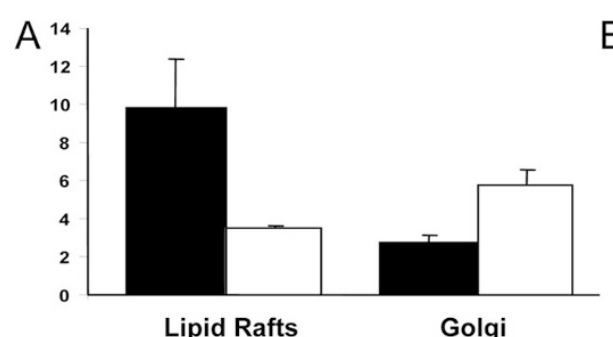

Lipid Rafts

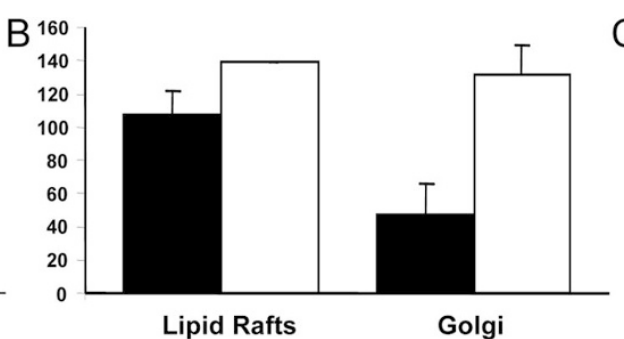

Lipid Rafts

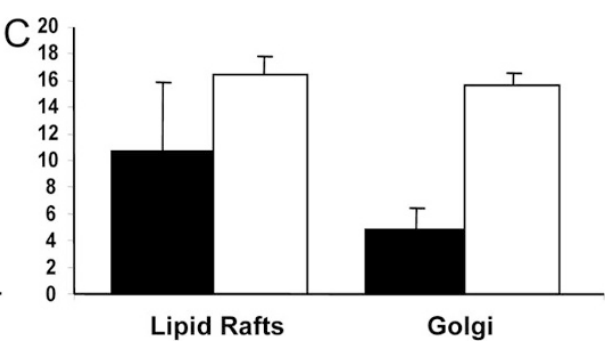

Lipid Rafts
Golgi

Figure 3. Metabolic galactose labeling of CLN3-deficient cells. (A) De novo GalCer; (B) de novo LacCer; (C) de novo globoside. De novo GalCer raft/Golgi ratio reverses in CLN3-deficient cells. Lipids are normalized to protein. Data are from three experiments, Normal human lymphoblasts ( $\square$ ); CLN3-deficient cells ( $\square$ ).

galactose-labeling, subcellular fractionation, lipid extraction, and TLC (Fig. 3). De novo GalCer is less in LR and more in Golgi fractions of CLN3-deficient lymphoblasts. Newly synthesized GalCer fails to reach rafts of CLN3-deficient cells supporting a trafficking defect. Lactosylceramide/Globoside de novo synthesis rates are elevated in CLN3-deficient compared with normal rafts, compensating for low de novo GalCer, and insuring stability (Fig. 3). All changes were statistically significant $(p<0.01$ for GalCer and $p \leq 0.05$ for LacCer and Globoside LR differences).

Mass measurement of glycosphingolipids from LR/ Golgi/ER is confirmed with organelle-specific markers: calreticulin (ER), GRASP65 (Golgi), and flotillin-1 (LRs). Sphingolipid composition of LRs/Golgi/ER is altered in JNCL cells (Fig. 4): GalCer is diminished in LRs, but accumulates in Golgi/ER. Changes are statistically significant with $p \leq 0.05$ for LR/Golgi/ER changes between normal and CLN3deficient cells. This corroborates confocal image results and agrees with de novo GalCer deposition in CLN3-deficient cells seen in Figure 3. Statistical significance reached $\leq 0.01$ for LacCer/globoside GlcCer/ceramide trihexoside changes in LR/Golgi/ER. Globoside mass was significantly higher in CLN3-deficient rafts than in normal LR.
GalCer distribution in subcellular fractions. Fractions 2/3 had the highest flotillin-1 expression and were designated as LR fractions. Fractions 4/5/6/7/8 were positive for GRASP65 corresponding to Golgi's. There was overlap between ER/ Golgi fractions $7 / 8$ as fractions 7/8/9 were also positive for ER calreticulin. GalCer was evenly distributed in ER (fractions $7 / 8$ ), and Golgi (fractions 4-8), and reached LR (fractions $2 / 3$ ) in normal cells. GalCer was retained in ER/Golgi of JNCL cells. GalCer was barely present in fraction 3 of LRs derived from JNCL cells (Fig. 5A). Transfecting JNCL cells with CLN3-containing vector results in reappearance of GalCer in fraction 3, compared with empty vector (Fig. $5 B$ ). GalCer antibody is specific for GalCer with no cross-reactivity to GlcCer/sulfatide.

Absence of GalCer. Galactosylceramide transferase (GCT) was knocked down by siRNA in normal cells and verified by quantitative RT-PCR. Confocal microscopy with fluorescent GalCer transfected cells versus cells transfected with scrambled siRNA, exhibit diminished growth (Fig. 5D). This finding suggests that reducing cell GalCer diminishes GalCer presence in PM, and this negatively impacts cell growth. Propidium iodide staining confirms increased apoptosis. Etoposide augments the effect of reduced GalCer on apoptosis (Fig.
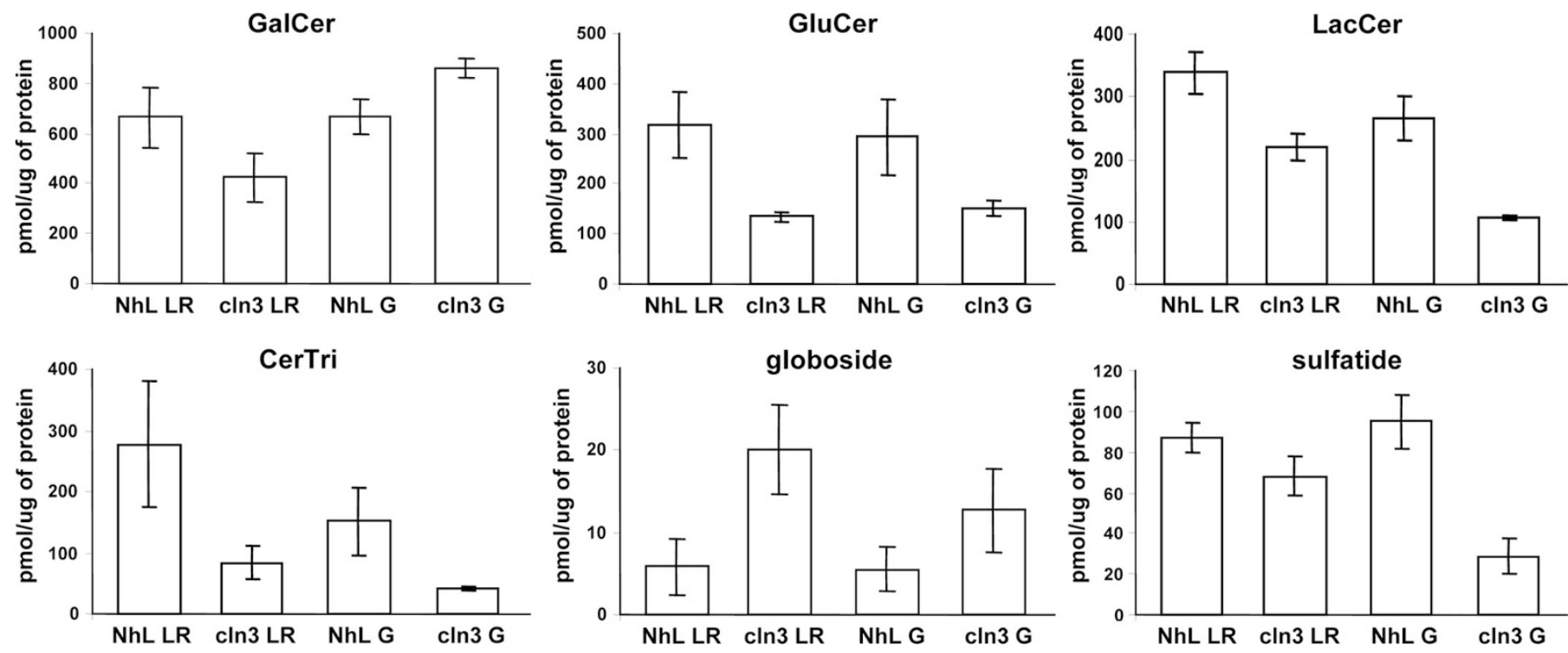

Figure 4. Mass measurement of glycosphingolipids in JNCL cells. GalCer raft/Golgi ratio reverses in CLN3-deficient cells compared with normal. GluCer, LacCer, and CerTri levels are lower in JNCL cells; globoside is elevated in LR and Golgi fraction the ImageQuant program. Lipids are normalized to protein. Results are average of three experiments; error bars represent SEM. NhL, normal human lymphoblasts ( $\square$ ); CLN3-deficient, JNCL cells ( $\square$ ). LR, lipid rafts; G, Golgi; GalCer, galactosylceramide; GlcCer, glycosylceramide; CerTri, ceramide trihexoside. 
A.

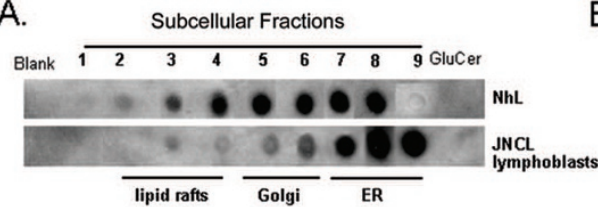

B.

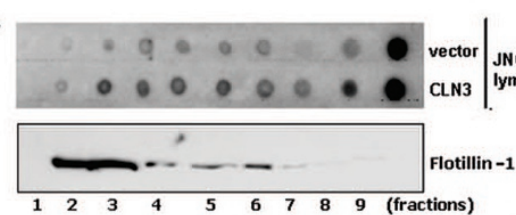

D. 1400

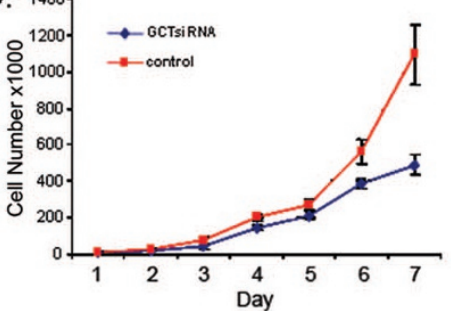

E.

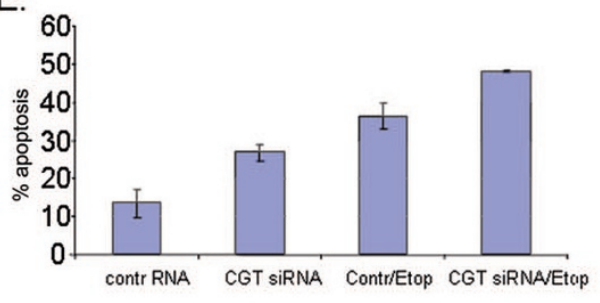

C.

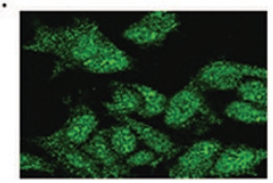

F. 140

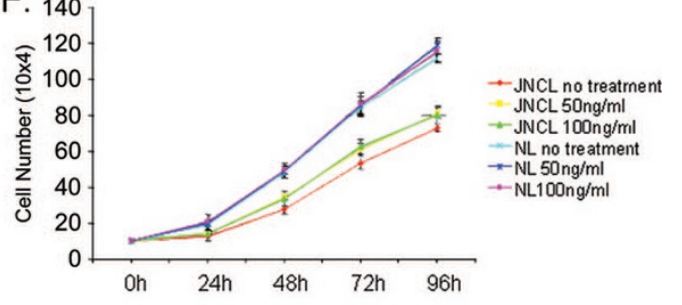

Figure 5. JNCL lymphoblast LR fractions are depleted of GalCer. (A) GalCer is abundant in ER and barely detectable in LR fractions from JNCL. (B) Reintroduction of CLN3p restores GalCer to rafts. Western blot (flotillin antibody): maximum flotillin coincides with LR fractions. (C) GCT knockdown with siRNA (right frame) $v s$. scrambled siRNA (left frame) causes reduced GalCer. (D) reduced cell growth of CGT siRNA vs. scrambled siRNA-transfected cells. (E) Propidium iodide staining demonstrates increased apoptosis of CGT siRNA vs. scrambled siRNA-transfected cells, which is enhanced by etoposide, and reduced by GalCer. $(F)$ GalCer supplementation improves JNCL cell growth. NhL, normal human lymphoblasts; JNCL, Juvenile Neuronal Ceroid Lipofuscinosis; ER, endoplasmic reticulum; GlcCer, glycosylceramide. LR fractions 2-3 are enriched in flotillin-1. Equal amounts/fraction are applied and probed with anti-GalCer antibody.
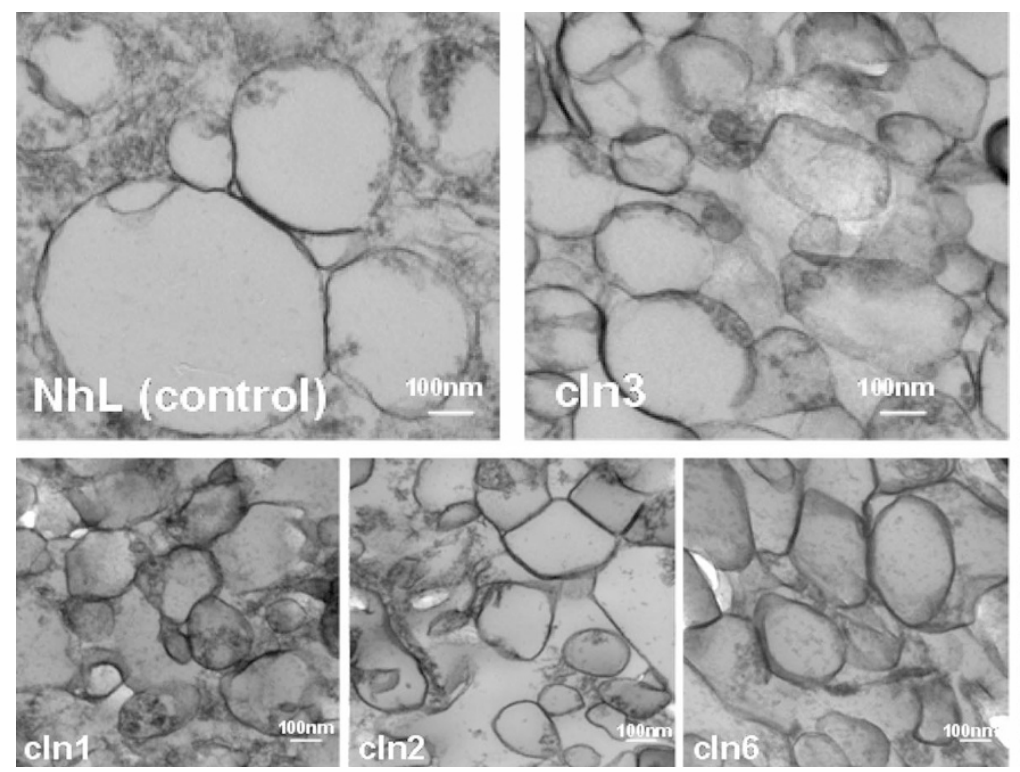
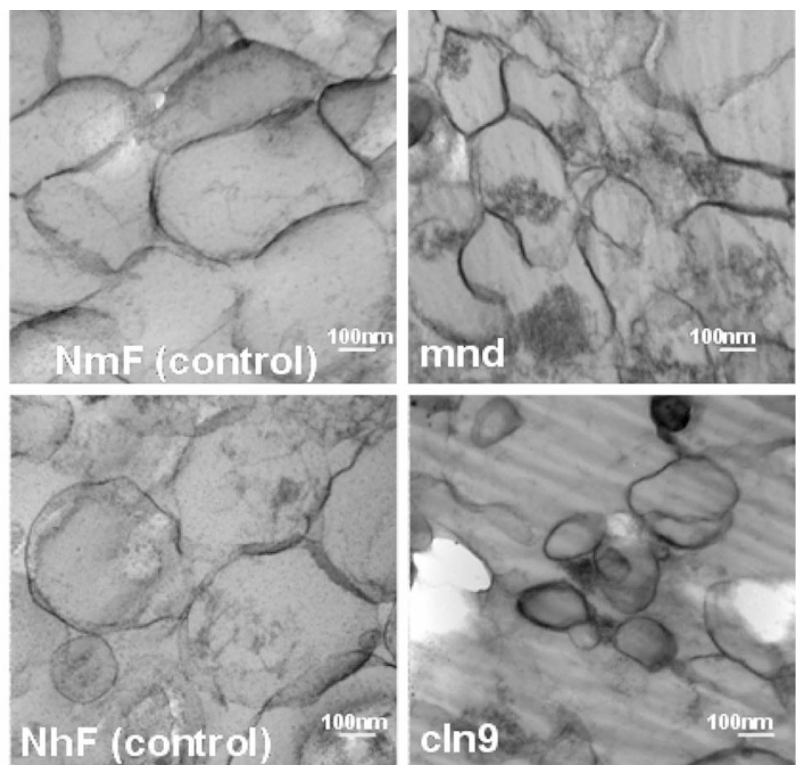

Figure 6. Transmission electron microscopy of CLN3-/CLN1-/CLN2-/CLN6-/CLN8-/CLN9-deficient cell LR. NhL, normal lymphoblasts; cln3, CLN3deficient lymphoblasts; cln1, CLN1-deficient lymphoblasts; cln2, CLN2-deficient lymphoblasts; cln6, CLN6-deficient lymphoblasts; NmF, normal mouse fibroblasts; mnd, CLN8-deficient fibroblasts; NhF, normal human fibroblasts; cln9, CLN9 deficient fibroblasts. (Magnification: 45,000×).

5E). Supplementing JNCL cells with GalCer at a concentration of $50 \mathrm{ng} / \mathrm{mL}$ restores growth (Fig. $5 F$ ). Doubling the concentration of GalCer had no effect.

Lipid raft morphology of NCL cells. A defined lipid stoichiometry is necessary for raft integrity. Because sphingolipid profiles of JNCL lipid rafts differ from normal, a change in raft morphology is anticipated. Raft fraction pellets were examined by transmission electron microscopy. Vesicular structure morphology for JNCL rafts was smaller in size $[50-200 \mathrm{~nm}$ $(\mathrm{nm})$ in JNCL compared with $300-600 \mathrm{~nm}$ in normal cells] and angular. Likewise, vesicular structures of rafts from CLN1-/CLN2-/CLN6-/CLN8-/CLN9-deficient cells are angular and smaller than normal (Fig. 6).

CLN1/CLN2/CLN6/CLN8 proteins bind lipids. The protein-lipid overlay assay with normal cell lysates and CLN1-/
CLN2-/CLN6-/CLN8-antibodies establishes that CLN6/ CLN8 proteins bind GalCer, CLN1/CLN2/CLN6p bind lysophosphatidic acid (LPA)/sulfatide and CLN8p binds ceramide (Fig. 7).

Sphingolipid mass measurement from CLN1-/CLN2-/ CLN6-/CLN8-/CLN9-deficient cellular fractions. Lipid composition of Golgi/LR of CLN1-/CLN2-/CLN6-/CLN8- and CLN9-deficient cell fractions were determined. Levels of GalCer/GlcCer/LacCer and ceramide trihexoside/globoside are altered in NCL cell lines (Fig. 8).

\section{DISCUSSION}

Sphingolipid/phospholipid biosynthesis occurs in ER/Golgi subcellular compartments. GlcCer is produced at the cytosolic 

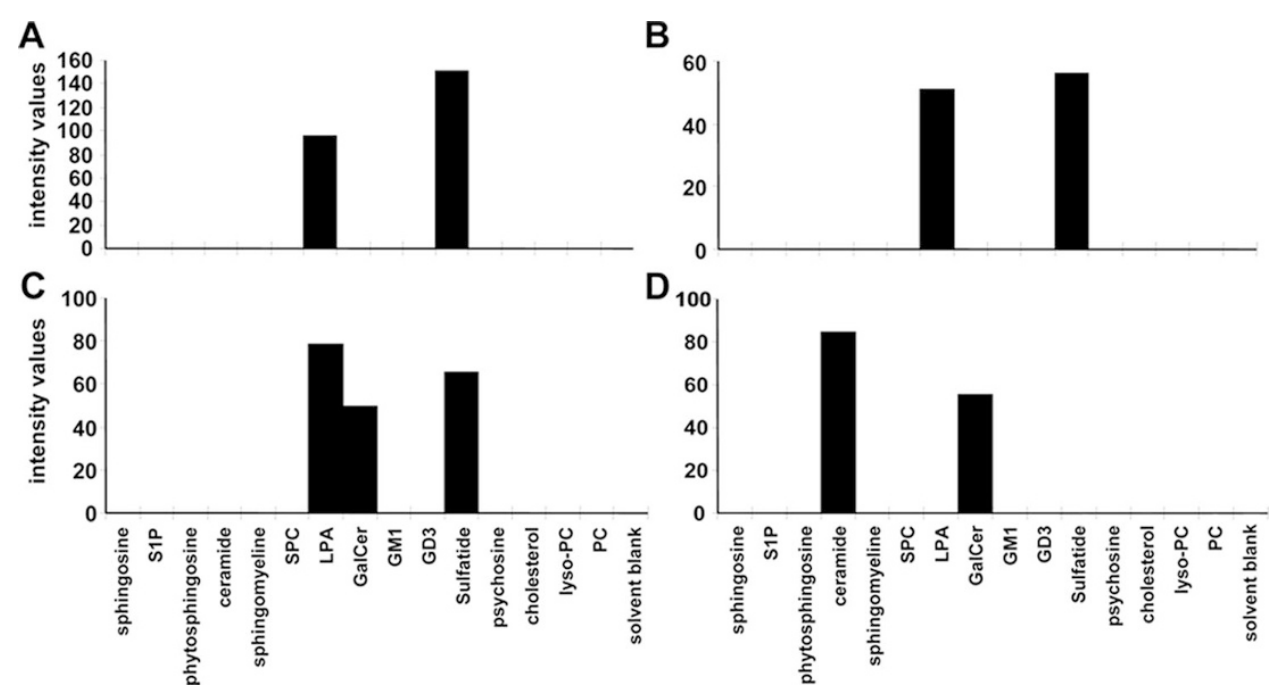

Figure 7. Lipid binding to $(A) \mathrm{CLN} 1,(B)$ CLN2, $(C)$ CLN6, and (D) CLN8 proteins. Lipids- $x$ axis; intensity values for relative lipid/NCL protein-binding- $y$ axis. Intensity calculated using the ImageJ program as described in Figure 2. Results were reproduced twice.
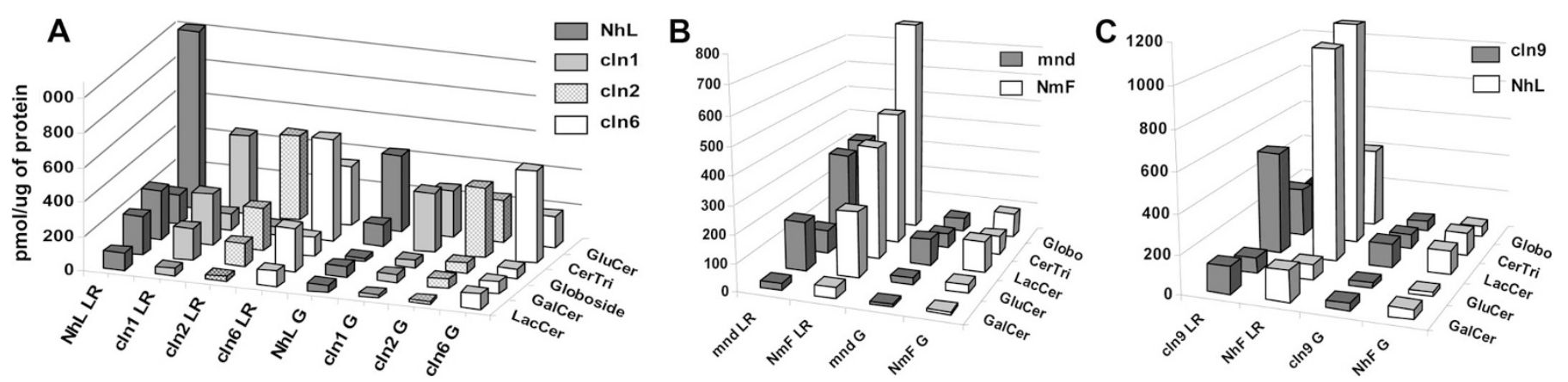

Figure 8. Raft sphingolipid composition of (A) CLN1-, CLN2-, CLN6-deficient lymphoblasts, and (B) CLN8- and (C) CLN9-deficient fibroblasts. Quantification is by the ImageQuant program (lipids normalized to protein). Results were reproduced three times.

side of Golgi (26) and translocates to lumen where it converts to LacCer and other complex glycosphingolipids. GalCer synthesis occurs in the ER lumen (27). Intracellular translocation of lipids from site of synthesis to final destination is mediated via specific transporters. Distribution of lipid species between inner/outer membrane leaflets is uneven. This gradient influences curvature/protein association and activity. Lipid transporting proteins, or flippases, are required for efficient transbilayer lipid movement (28). The P-glycoprotein encoded by the multidrug resistance gene, mdr1, transports GlcCer. Glycolipid transfer proteins (GLTP) are specific for neutral glycosphingolipids/gangliosides and intermembrane transfer of GalCer. The structures of GlcCer-GLTP/Lactosylceramide (LacCer)-GLTP/ganglioside GM3 or GM3-GLTP are characterized (29,30). The ceramide transporter CERT mediates ATP-dependent translocation of ceramide from ER to Golgi in a nonvesicular manner (31). The ABC (ATP-Binding Cassette) transporters couple ATP hydrolysis energy to import/ export of lipids/amino acids/ions/sugars/proteins, and drugs. Loss of function may cause cystic fibrosis, retinal defects, neurologic disease, and aberrant lipid/cholesterol distribution (32). Some transporters are designated as the multidrug resistance $\mathrm{ABC}$ (MDR-ABC) family, providing resistance to anticancer drugs. ER comes into close proximity with mitochondria/PM/trans-Golgi network/endosomes/lysosomes and phagosomes (33). Contact sites are bridgeable by a single protein forming intracellular communication facilitating rapid/ energy-efficient trafficking of lipids/other small molecules in/ out of the ER (34).

This study is the first linking a Batten protein to transport of a sphingolipid. GalCer, like CLN3p, localizes to rafts/Golgi/ early recycling endosomes. GalCer localizes to Golgi and minimally to recycling endosomes, but not to rafts in JNCL cells. Binding of WT CLN3p/GalCer and lack of binding between mutant CLN3/GalCer is demonstrated. Subcellular distribution of de novo GalCer/other sphingolipids is different in JNCL versus normal cells. Accumulation of GalCer in ER/Golgi with reduction of GalCer in rafts of CLN3-deficient cells supports that CLN3p is necessary for GalCer transport to rafts. Reintroduction of CLN3p restores GalCer to the lower LR fraction in JNCL cells. Correct stoichiometry of rafts is critical for proper functioning. Mass measurements of sphingolipids confirm that GalCer is diminished in rafts and abundant in ER/Golgi of JNCL cells. This correlates with immunocytochemical data demonstrating retention of mutant CLN3p/GalCer in Golgi, and confirms a defect in GalCer transport from ER/Golgi to rafts in JNCL. Size of vesicular structures in JNCL is smaller compared with healthy cells. Perturbations in sphingolipid levels are mirrored by a changed raft morphology. Normal cell vesicular structure size here is comparable with values in the literature reported for fibrobalsts and lymphoblasts (35). Reducing cell GalCer content by GCT siRNA 
diminishes GalCer levels in PM, negatively impacting cell growth, confirming the biologic significance of GalCer in rafts. We propose that CLN3 binds GalCer, extracts it from ER/Golgi carrying it to rafts. Failure to do so leads to a LR GalCer deficit in JNCL and results in multiple biologic aberrations.

GalCer LR content is important for healthy neurons/ oligodendrocytes. GalCer/ sulfatide are major components of myelin/oligodendrocyte PM (36) and of neuronal axons. GalCer is involved in Schwann cell-axon interactions, oligodendrocyte differentiation, and transduction of signals across the myelin membrane $(34,36)$.

In JNCL mutant CLN3p with ineffective GalCer binding fails to traffic to PM. Altered binding of mutant CLN3p to GalCer leads to failure of transport to rafts. This may cause failed targeting to rafts, inefficient GalCer delivery and perturbations in GalCer/ceramide/globoside, and GlcCer/ ceramide trihexoside in rafts. This alters physicochemical properties/structure, impairs raft function and enhances apoptosis by caspase- 8 activation. In fact, Hobert has reported decreased buoyancy of JNCL brain lipid rafts compared with controls (37). Isolated effects of decreased GalCer on growth/ apoptosis may explain neuronal/photoreceptor death in JNCL. Correction of JNCL cell growth by GalCer indicates GalCer retrograde trafficking is intact. This lays the groundwork for development of GalCer supplementation as treatment for JNCL. The work uncovers a novel mechanism for neurodegeneration. NCL complementation groups regarding growth/ apoptosis, coimmunoprecipitation of NCL proteins and colocalization to subcellular compartments are described (38). Binding of CLN1/CLN2/CLN6/CLN8p to sulfatide/GalCer/LPA/ ceramide, and raft sphingolipid content/morphology from cells deficient in these proteins suggest lipid-binding/trafficking defects and raft sphingolipid content/morphology are common themes for NCL. Manipulation of sphingolipid content or intracellular transport may provide avenues for therapy.

Acknowledgments. The authors thank the affected children and their families for donating samples. Their constant encouragement and unwavering support made this work possible.

\section{REFERENCES}

1. Boustany R-M 1996 Batten disease or neuronal ceroid lipofuscinosis. In: Moser H (ed) Handbook of Clinical Neurology, Neurodystrophies and Neurolipidoses, Vol. 66. Elsevier, New York, pp 671-900

2. Persaud-Sawin DA, VanDongen A, Boustany RM 2002 Motifs within the CLN3 protein: modulation of cell growth rates and apoptosis. Hum Mol Genet 11:2129-2142

3. Mao Q, Foster B, Xia H, Davidson BL 2003 Membrane topology of CLN3, the protein underlying Batten disease. FEBS Lett 541:40-46

4. Dhar S, Bitting RL, Rylova SN, Jansen PJ, Lockhart E, Koeberl DD, Amalfitano A, Boustany RM 2002 Flupirtine blocks apoptosis in Batten patient lymphoblasts and in human post-mitotic CLN3 and CLN2-deficient neurons. Ann Neurol 51:448-466

5. Cao Y, Espinola JA, Fossale E, Massey AC, Cuervo AM, MacDonald ME, Cotman SL 2006 Autophagy is disrupted in a knock-in mouse model of juvenile neuronal ceroid lipofuscinosis. J Biol Chem 281:20483-20493

6. Rylova SN, Amalfitano A, Persaud-Sawin DA, Guo W-X, Chang J, Jansen PJ, Proia AD, Boustany R-M 2002 The CLN3 gene is a novel molecular target for cancer drug discovery. Cancer Res 62:801-808

7. Kremmidiotis G, Lensink IL, Bilton RL, Woollatt E, Chataway TK, Sutherland GR, Callen DF 1999 The Batten disease gene product (CLN3p) is a Golgi integral membrane protein. Hum Mol Genet 8:523-531

8. Katz ML, Shibuya H, Liu PC, Kaur S, Gao CL, Johnson GS 1999 A mouse knock out model for Juvenile Neuronal Ceroid Lipofuscinosis (Batten Disease). J Neurosci Res 57:551-556
9. Luiro K, Kopra O, Lehtovirta M, Jalanko A 2001 CLN3 protein is targeted to neuronal synapses but excluded from synaptic vesicles: new clues to Batten disease. Hum Mol Genet 10:2123-2131

10. Margraf LR, Boriack RL, Routheut AA, Cuppen I, Alhilali L, Bennett CJ, Bennett MJ 1999 Tissue expression and subcellular localization of CLN3, the Batten disease protein. Mol Genet Metab 66:283-289

11. Persaud-Sawin DA, McNamara JO II, Rylova S, Vandongen A, Boustany RM 2004 A galactosylceramide binding domain is involved in trafficking of CLN3 from Golgi to rafts via recycling endosomes. Pediatr Res 56:449-463

12. Mahfoud R, Garmy N, Maresca M, Yahi N, Puigserver A, Fantini J 2002 Identification of a common sphingolipid-binding domain in Alzheimer, prion, and HIV-1 proteins. J Biol Chem 277:11292-11296

13. Huang Q, Shen HM, Shui G, Wenk MR, Ong CN 2006 Emodin inhibits tumor cell adhesion through disruption of the membrane lipid Raft-associated integrin signaling pathway. Cancer Res 66:5807-5815

14. Miyaji M, Jin ZX, Yamaoka S, Amakawa R, Fukuhara S, Sato SB, Kobayashi T, Domae N, Mimori T, Bloom ET, Okazaki T, Umehara H 2005 Role of membrane sphingomyelin and ceramide in platform formation for Fas-mediated apoptosis. J Exp Med 202:249-259

15. Gombos I, Kiss E, Detre C, Laszlo G, Matko J 2006 Cholesterol and sphingolipids as lipid organizers of the immune cells' plasma membrane: their impact on the functions of MHC molecules, effector T-lymphocytes and T-cell death. Immunol Lett 104:59-69

16. Brugger B, Glass B, Haberkant P, Leibrecht I, Wieland FT, Krausslich HG 2006 The HIV lipidome: a raft with an unusual composition. Proc Natl Acad Sci USA 103:26412646

17. Zaas DW, Duncan M, Rae Wright J, Abraham SN 2005 The role of lipid rafts in the pathogenesis of bacterial infections. Biochim Biophys Acta 1746:305-313

18. Hering H, Lin CC, Sheng M 2003 Lipid rafts in the maintenance of synapses, dendritic spines, and surface AMPA receptor stability. J Neurosci 23:3262-3271

19. London E, Brown DA 2000 Insolubility of lipids in triton X-100: physical origin and relationship to sphingolipid/cholesterol membrane domains (rafts). Biochim Biophys Acta 1508:182-195

20. Kilkus J, Goswami R, Testai FD, Dawson G 2003 Ceramide in rafts (detergent-insoluble fraction) mediates cell death in neurotumor cell lines. J Neurosci Res 72:65-75

21. Pullarkat RK, Morris GN 1997 Farnesylation of Batten disease CLN3 protein Neuropediatrics 28:42-44

22. Persaud-Sawin DA, Boustany R-M 2005 Cell death pathways in Batten disease. Apoptosis 10:973-989

23. Narayan SB, Rakheja D, Tan L, Pastor JV Bennett MJ 2006 CLN3P, the Batten's disease protein, is a novel palmitoyl-protein Delta-9 desaturase. Ann Neurol 60:570-577

24. Sakurai A, Okamoto K, Fujita Y, Nakazato Y, Wakabayashi K, Takahashi H, Gonatas NK 2000 Fragmentation of the Golgi apparatus of the ballooned neurons in patients with corticobasal degeneration and Creutzfeldt-Jakob disease. Acta Neuropathol 100:270-274

25. Fujita Y, Okamoto K, Sakurai A, Gonatas NK, Hirano A 2000 Fragmentation of the Golgi apparatus of the anterior horn cells in patients with familial amyotrophic lateral sclerosis with SOD1 mutations and posterior column involvement. J Neurol Sci 174:137-140

26. Jeckel D, Karrenbauer A, Burger KN, van Meer G, Wieland F 1992 Glucosylceramide is synthesized at the cytosolic surface of various Golgi subfractions. J Cell Biol 117:259-267

27. Sprong H, Degroote S, Nilsson T, Kawakita M, Ishida N, van der Sluijs P, van Meer G 2003 Association of the Golgi UDP-galactose transporter with UDP-galactose: ceramide galactosyltransferase allows UDP-galactose import in the endoplasmic reticulum. Mol Biol Cell 14:3482-3493

28. Eckford PD, Sharom FJ 2005 The reconstituted P-glycoprotein multidrug transporter is a flippase for glucosylceramide and other simple glycosphingolipids. Biochem J 389:517-526

29. Gammon CM, Vaswani KK, Ledeen RW 1987 Isolation of two glycolipid transfer proteins from bovine brain: reactivity toward gangliosides and neutral glycosphingolipids. Biochemistry 26:6239-6243

30. Rao CS, Chung T, Pike HM, Brown RE 2005 Glycolipid transfer protein interaction with bilayer vesicles: modulation by changing lipid composition. Biophys $\mathrm{J}$ 89:4017-4028

31. Hanada K, Kumagai K, Yasuda S, Miura Y, Kawano M, Fukasawa M, Nishijima M 2003 Molecular machinery for non-vesicular trafficking of ceramide. Nature 426:803-809

32. Gottesman MM, Ambudkar SV 2001 Overview: ABC transporters and human disease. J Bioenerg Biomembr 33:453-458

33. Levine TP 2004 Membrane contact sites, a network for short-range intracellular communication. Trends Cell Biol 9:483-490

34. Boggs JM, Wang H 2004 Co-clustering of galactosylceramide and membrane proteins in oligodendrocyte membranes on interaction with polyvalent carbohydrate and prevention by an intact cytoskeleton. J Neurosci Res 76:342-355

35. de Gassart A, Geminard C, Fevrier B, Raposo G, Vidal M 2003 Lipid raft-associated protein sorting in exosomes. Blood 102:4336-4344

36. Hirahara Y, Bansal R, Honke K, Ikenaka K, Wada Y 2004 Sulfatide is a negative regulator of oligodendrocyte differentiation: development in sulfatide-null mice. Glia 45:269-277

37. Hobert JA, Dawson G 2007 A novel role of the Batten disease gene CLN3: association with BMP synthesis. Biochem Biophys Res Commun 358:111-116

38. Persaud-Sawin DA, Mousallem T, Wang CH, Zucker A, Kominami E, Boustany R-M N 2007 Neuronal ceroid lipofuscinosis: a common pathway? Pediatr Res 61:146-152 